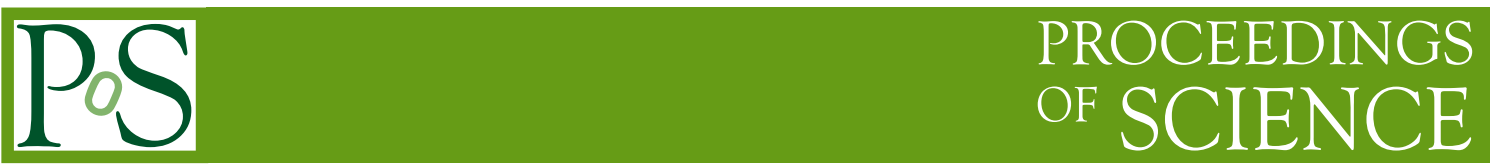

\title{
Supernova Legacy Survey 3-years data sample
}

\author{
Delphine HARDIN, on behalf of the Supernova Legacy Survey (SNLS) * \\ LPNHE, Université Pierre et Marie Curie, Paris, France \\ E-mail: hardinein2p3.fr
}

\begin{abstract}
We present the recent cosmology results from the Supernova Legacy Survey (SNLS) 3 years analysis. Using 242 high redshift type Ia supernovae discovered and measured during the first 3 years of the Supernova Legacy Survey, together with other mostly nearby supernovae samples, we measure a dark energy equation of state parameter $w$ consistent with a cosmological constant. The sytematic uncertainties are approximately equal to the statistical uncertainties. Combining the supernovae data with cosmological microwave background (WMAP7) and baryonic acoustic oscillations (SDSS) measurements, we obtain a measurement of the dark energy equation of state $w=-1.068$ with a precision of 0.08 .
\end{abstract}

XXIst International Europhysics Conference on High Energy Physics

21-27 July 2011

Grenoble, Rhônes Alpes France

${ }^{*}$ Speaker. 


\section{Introduction}

The Nobel Prize in Physics 2011 was awarded jointly to Saul Perlmutter, Brian P. Schmidt and Adam G. Riess for the discovery, in the late 90's, of the accelerating expansion of the Universe through observations of distant supernovae[1, 2]. The Supernova Cosmology Project and the High $\mathrm{Z}$ Team collected data on type Ia supernovae discovered and monitored with CCDs camera on 4-m class telescope, and, using them as standardized candles, proved that the universe expansion had stop decelerating 5 Gyr ago ${ }^{1}$.

Since then, other cosmological probes - the cosmic microwave background (CMB) temperature fluctuations, the baryon acoustic oscillations (BAO) imprinted in the galaxies spatial distribution, weak gravitational lensing mapping dark matter clustering etc. - have provided new evidence for the presence of a dark energy component opposing gravity and accounting for $75 \%$ of the total universe energy density. However, the physical origin of cosmic acceleration remains mysterious.

The new generation supernova surveys have permitted to increase the number of SNeIa distance measurements up to $\sim 1000$, while improving their quality and their homogeneity. Combining the different cosmological probes, it is now possible to adress the question of the dark energy nature, by measuring its equation of state parameter $: w=p_{X} / \rho_{X}$.

The Supernovae Legacy Survey is a 5 year program which aims at measuring the dark energy equation equation of state $w$ in combination with other measurements to better than 0.10 . With the reduction of statistical errors, sytematics errors have become the limiting factor and as such the key issue : paying special attention to include and limit thoroughly any systematics effects is mandatory.

\section{Expansion history and the universe content}

In an expanding isotropic and homogeneous universe, distances between galaxies at rest scale as $d \propto a(t)$. The light emitted by one observer at time $t$ and observed by another at later time $t_{0}$ is observed to be redshifted, by a factor $1+z=a\left(t_{0}\right) / a(t)$, directly related to the scale factor $a(t)$. The expansion dynamics is governed through the Einstein equations by the universe energy content. Matter, with a present day reduced density $\Omega_{M}=\rho_{M}\left(t_{0}\right) / \rho_{\text {crit }}\left(t_{0}\right)$ - the critical density corresponds to a flat geometry for the universe - decelerates the universe expansion. An accelerating expansion could be explained by the existence of a second energy component, a perfect fluid $X$, with a repulsive equation of state $w=p_{X} / \rho_{X}<-1 / 3$ and a corresponding reduced density $\Omega_{X}$ : the dark energy. Mapping the expansion history $a(t)$ makes possible to measure the cosmological parameters $\Omega_{M}, \Omega_{X}$, and $w$.

The nature of the dark energy remains an enigma. It could be a constant energy component, such as the cosmological constant $\Lambda$ originally postulated by Einstein, or, formally equivalent, the vacuum energy of particle physics, in these cases $w=-1$. Another possibility is that the dark energy be a dynamical fluid, whith a time variating equation of state $w(z)-$ or $w(a)$. An alternative explanation would require to modify the General Relativity at cosmologically large scales, or invoke inhomogeneities inducing apparent acceleration (the "back-reaction").

\footnotetext{
${ }^{1}$ See e.g. (Frieman, 2009)[3] for a review.
} 


\section{Type Ia supernovae : standard candles as cosmological probes}

For an object of intrinsic luminosity $L$, the flux $f$ measured by the observer defines the luminosity distance in term of the usual inverse square law : $f=L /\left(4 \pi d_{L}^{2}\right)$. The dependency of $d_{L}$ on the redshift $z$ (the Hubble diagram) is related to the integrated expansion history along the line of sight, and as such to the energy content of the universe and the aforementionned cosmological parameters :

$$
d_{L} \equiv \sqrt{f / 4 \pi L}=c z / H_{0} \times \mathscr{D}\left(z ; \Omega_{M}, \Omega_{X}, w\right)
$$

As one measure fluxes and not luminosities, standard candles are of particular importance : because these objects are considered to have the same luminosity $L$, they can be used to measure relative distances. This way, it is possible to constrain the cosmological parameters without having to know the luminosity $L$, nor the Hubble constant $H_{0}$ values.

Type Ia supernovae ( $\mathrm{SNe}$ ) are cosmic explosions that display an impressive homogeneity. These rare (1 per galaxy per millenium) and bright events, observable at cosmological distances, are thought to arise from the explosion of a white dwarf accreting matter from a companion and reaching the stability Chandrasekhar mass limit. The companion could be an evolved main sequence star, a red giant or a white dwarf. They are easily identified using spectroscopy, as they exhibit strong absorption features at the time of their peak luminosity.

With a $40 \%$ dispersion of their peak luminosity measured in the Johnson B band, they qualify as standard candles. They also exhibit a correlation bewteen their peak luminosity and the time evolution behaviour of their lightcurve, as well as with their restframe colors. By taking into account these empirical relations - acknowledged as the brighter-slower and the brighter-bluer relations - , the observed dispersion is reduced to $\sim 10 \%$. This results in a dispersion of $\sim 5 \%$ for the estimated distance : they are standardized candles.

In order to estimate the cosmological parameters, we will be comparing the restframe peak fluxes (e.g. in the restframe Johnson B band) of SNe Ia exploding at different redshifts (Eq. 3.1). This requires to observe them in different filters, which intercalibration must then be precisely known. To evaluate the restframe Johnson B band value at peak, one needs to interpolate the fluxes measurement between several different filters and also at different dates : for this we use a time dependent spectrophometric model of the SN Ia emission, $\phi(\lambda, t)$, that we construct empirically using spectrophometric data of nearby and distant SNe Ia.

The relationship between distance $d$, flux $f$ and luminosity $L$ translates simply in the logarithmic scales of the magnitudes, involving the corresponding quantities of distance modulus $\mu$, apparent magnitude $m$ and absolute magnitude $\mathscr{M}$ :

$$
\mu=m_{B}^{\star}-\mathscr{M}, \mathscr{M}=M_{B}-\alpha \times \text { shape }+\beta \times \text { color }
$$

For each SN are estimated the three quantities $m_{B}^{\star}$, corresponding to the peak B flux, the shape of its lightcurve, and its color at peak. The absolute magnitude of the $\mathrm{SN}$ is parametrized so that $M_{B}$ (fully degenerated with $H_{0}^{2}$ ) corresponds to the luminosity of the (shape=0, color $=0$ ) standard SN Ia, and $\alpha$ and $\beta$ empirically account for the linear corrections corresponding respectively to the brighter-slower and the brighter-bluer relations. The cosmological parameters are evaluated

\footnotetext{
${ }^{2}$ We fix $H_{0}=70 \mathrm{~km} / \mathrm{s} / \mathrm{Mpc}$.
} 
through a $\chi^{2}$ fit where $\mu$ is compared to its predicted value $\mu_{\text {cosmo }}(z ; \vec{\theta}), \vec{\theta}$ beeing the parameters describing the cosmological model. $M_{B}, \alpha$ and $\beta$ are fitted on the Hubble diagram along with the cosmological parameters.

\section{The Supernova Legacy Survey}

The Supernova Legacy Survey experimental setup and strategy have been designed so as to obtain sufficient quality data and to meet the necessary requirements to control the systematics.

Using the 1 square degree imager Megacam[4] mounted on the 3.6-m Canadian-France-Hawai Telescope at Mauna Kea (Hawaii), we obtain a survey deep enough to reduce the Malmquist bias that affects all flux limited surveys[6].

Both supernovae discovery and photometry are carried out with one instrument. We can thus devoid all the necessary time to the thorough understanding and the calibration of the instrument[5].

SNLS is a rolling search i.e. we repeat observations of the same 4 fields, enabling the followup of the already discovered SN and the detection of newly exploded SN at the same time. It is thus possible to go back in our image data base to recover early, pre-discovery SN photometry. This strategy permits to obtain well sampled lightcurve so as to measure precisely of $m_{B}^{\star}$ and the lightcurve shape.

The four griz filters make it possible to measure the B restframe flux from $\mathrm{z}=0.1$ to $\mathrm{z}=1$., and also estimate precisely the restframe U-B and B-V colors of the SNe.

By observing 40 nights a year during 5 years (the survey ended in August, 2008), we obtained $\sim 450 \mathrm{SNe}$ Ia. All were spectroscopically identified on 10-m class telescopes[7, 9, 10, 11], which allows to limit the non Ia contamination of the sample.

Finally, deep SN-free images stacks were built, to estimate the SNe host galaxy colors, enabling to caracterize the SN environment.

\section{SNLS-3 years data Analysis}

The constraints and systematic uncertainties from the SNLS-3 data are fully detailed in Conley et al.[12]. We present here the SN sample, and some of the different steps involved in the data analysis.

SNLS is mainly a European and Canadian project : all the analysis steps were performed with independent pipelines on each side of the atlantic ocean. Calibration, the spectro-photometric model have been improved since the SNLS-1 year results. We also reckon with the host galaxy nature influence.

Finally, when estimating the cosmological parameters, we fully take into account the sytematics uncertainties as well as the statistical ones by incorporating them in the covariance matrix that enter the $\chi^{2}$ minimization procedure. Publishing this full covariance matrix makes it possible to other authors to exploit the SN data without loss of information. 


\subsection{The supernovae sample}

To obtain precise cosmological measurement requires a long enough lever-arm in redshift in the Hubble diagram (Eq. 3.1). We must then add to the SNLS sample complementary supernovae sample coming from external surveys.

The supernovae sample consists of 472 SNe Ia : 123 nearby supernovae, 93 at intermediate redshift form the Sloan Digitized Sky Survey (SDSS) supernovae search, 242 from SNLS and 14 Hubble Space Telescope (HST) supernovae.

The nearby sample at $z \sim 0$ are gathered from various sources, mainly the Calan/Tololo[13], the CfAI-II-III[14] and the CSP[15] searches. The photometry of one third of this sample is expressed in the Landolt system, to which the Megacam magnitude system must be tied : this crosscalibration requirement induces the main systematic effect in this analysis.

At $z>1$, ground observations are difficult, and the HST sample of 14 SNe Ia at $z=0.7-1.4$ complements the high redshift part of the Hubble diagram.

Finally, we add 146 SNe Ia at intermediate redshift $z<0.4$ from the SDSS Supernova Survey. This component of the SDSS-II survey carried out repeat imaging of a 300 square degree southern equatorial stripe using a dedicated 2.5-meter telescope in drifts scan mode at Apache Point Observatory, New Mexico. They discovered and measured about 500 spectroscopically confirmed SNe Ia. The SDSS filter system has been thouroughly studied and is very similar to the Megacam system : as a consequence the intercalibration is not as problematic as with the nearby sample.

\subsection{Calibration}

The calibration procedure of the SNLS data achieved an accuracy of a $1 \%$ precision[5]. It consists in two steps : the observations are first standardized onto some magnitude system, using a catalog of standard stars of known magnitudes. Then the standard system magnitudes are converted into absolute fluxes : for this we rely on a reference star, of known magnitudes and spectral energy density (SED). Both SNLS and SDSS survey selected a red reference star measured by the HST CALSPEC[16] calibration program.

To achieve the required precision, the spatial non-uniformities of the imager were mapped using dithered observation of dense stellar fields. Because part of the external low-z SNe sample is calibrated against the Landolt UBVRI system, the Megacam griz system has to be anchored to the Landolt system. The uncertainties in the Landolt magnitudes of our reference star BD $17^{\circ} 4708$ are the largest single identified sytematic uncertainty in our current analysis.

\subsection{The spectro-photometric model : SALT2 \& SIFTO}

To obtain for each SN the peak restframe B band magnitude and its lightcurve shape and color, we make use of two independent lightcurve fitters, SIFTO[17] and SALT2[18]. The SALT2 model for the rest-frame flux parametrization may be written as :

$$
\phi(\lambda, t)=X_{0} \times\left[M_{0}(\lambda, t)+X_{1} \times M_{1}(\lambda, t)\right] \exp (C C L(\lambda))
$$

$M_{0}$ is the mean spectrum and the corresponding parameter $X_{0}$ is the flux normalisation. $M_{1}$ describes the main variability of the $\mathrm{SNe}$ Ia and happens to naturally reproduce a brighter-slower 
relation : $X_{1}$ is thus equivallent to a lightcurve shape parameter. $C$ corresponds by construction to a color, and the color law CL encodes the corresponding variation of the model.

$M_{0}, M_{1}$ and CL are computed using a training sample of nearby and SNLS SNe Ia lightcurves and spectra. The SNe distances are not used, which makes it possible to use nearby as well as distant $\mathrm{SNe}$ for the training. As the U band data from nearby $\mathrm{SNe}$ turned out to be problematic, we used u' measurement of nearby data when available, and we especially rely on distant $\mathrm{SNe} g$ optical data, which sample the UV restframe at a redshift of $z \sim 0.4$.

No assumption were made on the color law $C L$ wavelength dependency nor its cause - wether it be due to intrinsic SNe Ia variation or to the reddening by dust somewhere along the line of sight : in the intergalactic medium, the host galaxy or a dust shell around the SN. As there is no a-priori knowledge of the dust properties, or its putative evolution with environment and/or redshift, no prior was set on the distribution of the $\mathrm{SNe}$ Ia C (color) parameter.

The color law is mathematically equivalent to an extinction law, but it does differ in the UV part from the dust extinction law as measured in the Milky Way Galaxy[19], and also the selective ratio corresponding to $\beta \sim 2.5-3$ in Eq. 3.2 is smaller than the MW value $R_{B} \sim 4$. This differences can be interpreted either as an unusual extinction occuring in the $\mathrm{SN}$ environnement, or an intrinsic color variation dominating the extinction effects.

In a nutshell, we make no assumption and let the SNe Ia data decide : on the range of their color value, on the selective ratio $\beta$ value, and on the color law CL wavelength dependency.

SIFTO model consists of a SED sequence, which is time dilated by a stretch factor depending on the wavelentgh. It does not contain an explicit color variation law but a linear color relation tailing the color $=\mathrm{B}-\mathrm{V}$ to the $\mathrm{U}-\mathrm{B}$ color, and the lightcurve shape.

Comparing the two fitters results permits to evaluate the uncertainties associated with the different choices involved in their design. As they perform equally well, we use the average of the two, and propagate the differences as systematic uncertainties.

\subsection{Host galaxy nature influence}

To address the question whether $M_{B}, \alpha$ and $\beta$ in Eq. 3.2 are universal parameters, and whether there is any dependence on the SN environnement, we undertook a photometric study of the SN host galaxies. Their ugriz fluxes were measured on deep stacked images free of SN light, and supplementary data from the WIRDS survey[20] in the IR part was added. The photometric data are fitted by templates spectral energy densities, using the redshift information from the SN : this permits to derive the restframe colors of the galaxy, and its intrinsic luminosity. Using SEDs computed with the population synthesis model PEGASE.2[21], one can recover the caracteristics of the synthetic model galaxy, such as its present star formation rate (SFR), and its stellar mass content.

The host galaxies properties are known[22, 23] to correlate with the SNe shape parameter : SNe Ia in red/high SFR/ low mass/faint galaxies are slower, and as a consequence, brighter. This could result from different evolutionary pathes leading to the explosion of the parent white dwarf[24, 25].

Although in massive galaxies the mean SN Ia is fainter, it has been recently brought to evidence $[26,27,28]$ that the standard (shape $=0$, color $=0$ ) SN Ia is in fact there slightly brighter, 
at a $4-\sigma$ significance : this is a subtle effect $-0.08 \mathrm{mag}$, or $8 \%$ - smaller than the shape or color corrections.

We take this dependency into account by splitting the sample at $M=10{ }^{10} M_{\odot}$ between low and high mass galaxies, and using two different $M_{B}$ values for each sub-sample. This leads to a significative improvement in the cosmological fits (at a $\sim 4 \sigma$ level) and also to a shift in the measured cosmology : for a flat universe, $\Omega_{M}$ value is shifted by an amount comparable to the statistical precision.

\subsection{Including the systematics}

The $\chi^{2}$ minimization procedure involves the residuals computed for each $\mathrm{SN} i: r_{i}=\mu_{i}-$ $\mu_{\text {cosmology }}\left(z_{i} ; \overrightarrow{\boldsymbol{\theta}}\right)$ and the associated covariance matrix $\mathbf{C}: \chi^{2}={ }^{t} r \mathbf{C}^{-1} r$. The covariance matrix $\mathbf{C}$ can be splitted in 3 terms : $\mathbf{C}=\mathbf{D}_{\text {stat }}+\mathbf{C}_{\text {stat }}+\mathbf{C}_{\text {sys }}$.

$\mathbf{D}_{\text {stat }}$ is a diagonal part dealing with purely statistical uncertainties, which includes the errors on the light curve parameters of each $\mathrm{SN}$ and on its redshift value, plus several additional terms: $\sigma_{\text {int }}$ to account for the intrinsic scatter of SNe Ia $-\sigma_{\text {int }}=0.07$ mag (or equivalently $7 \%$ ) for the SNLS sample; $\sigma_{\text {lensing }}=0.055 \times z$ to account for the gravitational lensing by foreground galaxies [29] ; $\sigma_{\text {host }}$ to account for the mis-classification of the SN host due to the host colors statistical errors.

As all the $\mathrm{SNe}$ shares the same spectro-photometric model used to estimate ( $m_{B}^{\star}$, shape,color), the statistical covariance is not diagonal and $\mathbf{C}_{\text {stat }} \neq 0$.

Finally, the $\mathbf{C}_{\text {syst }}$ part accounts for the systematic errors affecting the $\mathrm{SN}$ measurements i.e. the uncertainties that will not be reduced by increasing the sample size. For example, the uncertainty on one of the calibration zero point will affect for each SN the estimation of $\left(m_{B}^{\star}\right.$, color $)$, not only through the value of this SN photometric points, but also through the model, which is trained on many other $\mathrm{SNe}$ photometry. The most important term entering $\mathbf{C}_{\text {syst }}$, in terms of consequence on the cosmological parameters systematic uncertainties, is by far the calibration, especially the intercalibration of the different $\mathrm{SNe}$ sample.

\section{SNLS-3 years cosmological results}

The SNLS-3 cosmological results are presented in Conley et al.[12] and Sullivan et al.[30]. Including all identified systematic effects in the $\Omega_{M}-w$ plane assuming a flat universe is shown on Fig. 1 as the blue contours. They are consistent with a cosmological constant. Including the systematic nearly double the size of the uncertainty-"ellipse". Excluding the calibration sytematic reduces this increase down to $\sim 10 \%$.

The degeneracy along the $\Omega_{M}$ axis is lifted when constraints from other cosmological probes are added. The measurements of the CMB temperature fluctuation yields estimation for[31] : the acoustic scale $l_{A}=\pi\left(1+z_{*}\right) D_{A}\left(z_{*}\right) / r_{s}\left(z_{*}\right)$; the shift parameter $R$; the redshift at decoupling $z_{*}$ ; The imprint of the Baryon Acoustic Oscillation (BAO) in the galaxies correlation function at a given redshift $z$ yields a measurement of $r_{s}\left(z_{d}\right) / D_{V}(z)$ where $r_{s}\left(z_{d}\right)$ is the comoving sound horizon at the baryon drag epoch and $D_{V}(z)$ is the spherical average of the angular-diameter distance and the radial proper distance[32, 33].

Combining results from the Wilkinson Microwave Anisotropy Probe 7-years (WMAP7)[34] and from the SDSSS Data Release 7 BAO measurement[35] yield the "green" contours shown on 
Fig.1. Combining both contours which are almost orthogonal yields $w=-1.068_{-0.082}^{+0.080}-$ this result is nearly equivalent to fix $\Omega_{M}=0.27$ for the SNe Ia only contour.

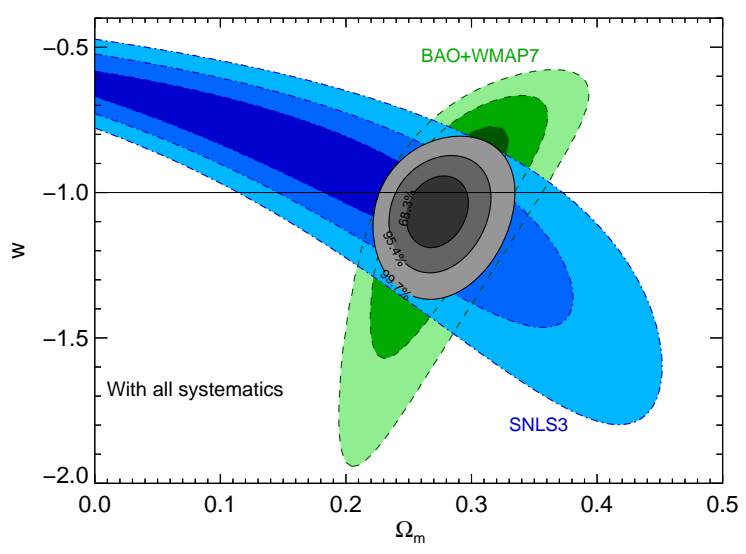

Figure 1: Confidence contours in the plane $\Omega_{M}-w$ obtained when fitting with the SNe Ia -only fit (in blue). A flat cosmology is assumed. Taking into account the systematics nearly double the size of the contour. The degeneracy along the $\Omega_{M}$ axis is lifted when adding the SDSS BAO and WMAP7 CMB constraints (in green).

\section{Conclusion}

We have combined the SNLS-3 SNe Ia data with baryon acoustic oscillation measurements from the Sloan Digital Sky Survey (SDSS) Data Release 7, and measurements of the cosmic microwave background power spectrum from the WMAP 7-year data. We obtain a most precise measurement of the dark energy equation of state consistent whith a cosmological constant.

The statistical uncertainties on the cosmological parameters are now exceeded by the systematics, the latter beeing dominated by the photometric calibration of the SN Ia fluxes.

The situation thus could change were the major contribution of the calibration to the systematics to be reduced. Indeed, because the SDSS filter system is similar to the Megacam system, and as low redshift sample observed in a very similar way will become available, there will be a possibility in the future to take full advantage of the inter-calibration improvements between supernovae sample.

In the near future, the SkyMapper[36] project will provide nearby SNe Ia at $z \sim 0.05$ discovered and observed with a similar technique than SNLS. Next generation surveys either groundbased such as the Large Synodic Survey Telescope (LSST) project or space-born such as the Wide Field Infrared Survey Telescope (WFIRST) or the EUCLID mission could bring thousands of distant SNe Ia up to $z \sim 1.5$. Providing an adapted strategy, they could adress the question of a time variating equation of state for the dark energy, $w(a)=w_{0}+(1-a) w_{a}[37]$.

\section{References}

[1] A. G. Riess et al., Astronomical Journal 116, 1009 (1998) 
[2] S. Perlmutter et al., Astrophysical Journal 517, 565 (1999)

[3] J. Frieman, "Lectures on Dark Energy and Cosmic Acceleration", arXiv:0904.1832

[4] O. Boulade et al., Instrument Design and Performance for Optical/Infrared Ground-based Telescopes, ed M.Iye \& A.F.M. Moorwood, Proc SPIE, 4841, 72

[5] N. Regnault et al., Astronomy and Astrophysics 506, 999 (2009)

[6] C. Perrett et al., Astronomical Journal 140, 518 (2010)

[7] D.A. Howell, et al., Astrophysical Journal 634, 1190 (2005)

[8] T.J. Bronder, et al., Astronomy and Astrophysics 477, 717 (2008)

[9] R.S. Ellis, et al., Astrophysical Journal 674, 51 (2008)

[10] C. Balland et al., Astrophysical Journal 507, 85 (2009)

[11] E. Walker et al., Monthly Notices of the Royal Atronomical Society 410, 1262 (2011)

[12] A. Conley et al., The Astrophysical Journal Supplement 192, 1 (2011)

[13] M. Hamuy et al., Astronomical Journal 112, 2408 (1996)

[14] M. Hicken et al., Astrophysical Journal 700, 331 (2009)

[15] C. Contreras et al., Astronomical Journal 139, 519 (2010)

[16] Bohlin, R.C. Astronomical Journal 111, 1743 (1996)

[17] A. Conley et al., Astronomical Journal 681, 482 (2008)

[18] J. Guy et al., Astronomy and Astrophysics 466, 11 (2007)

[19] J.A. Cardelli et al., Astrophysical Journal 345, 245 (1989)

[20] R. Bielby et al., in preparation

[21] D. Le Borgne et al., Astronomy and Astrophysics 386, 446 (2002)

[22] M. Hamuy et al., Astronomical Journal 120, 1479 (2000)

[23] M. Sullivan et al., Astrophysical Journal 648, 868 (2006)

[24] E. Scannapieco et al., Astronomical Journal 629, L85 (2005)

[25] F. Manucci et al., Monthly Notices of the Royal Atronomical Society 370, 773 (2006)

[26] P.L. Kelly et al., Astrophysical Journal 715, 743 (2010)

[27] H. Lampeitl et al., Astrophysical Journal 722, 766 (2010)

[28] M. Sullivan et al., Monthly Notices of the Royal Atronomical Society 406, 782 (2010)

[29] M. Jönsson et al., Monthly Notices of the Royal Atronomical Society 405, 535 (2010)

[30] M. Sullivan et al., submitted

[31] E. Komatsu et al., The Astrophysical Journal Supplement 180, 330 (2009)

[32] D. Eisentein et al., Astrophysical Journal 633, 560 (2005)

[33] C. Blake et al., arXiv:1105.2862

[34] E. Komatsu et al., The Astrophysical Journal Supplement 192, 18 (2011)

[35] W.J. Percival et al., Monthly Notices of the Royal Atronomical Society 401, 2148 (2010)

[36] S.C. Keller et al., Publications of the Astronomical Society of Australia 24, 1 (2007).

[37] P. Astier et al., Astronomy and Astrophysics 525, 7 (2011) 